\title{
Zur Textbehandlung
}

$W^{m}=$ Eigenhändige Randbemerkungen von Wilamowitz.

Im Text erscheinen diese Bemerkungen eingeschlossen in gerade eckige Klammern: $\left[\ldots W^{\mathrm{m}}\right]$.

$[\ldots]$, gegebenenfalls $[\ldots]$, schräge Schrift in schrägen eckigen Klammern $=$ Bemerkungen und Zusätze der Bearbeiter.

Änderungen am Text.

Geăndert sind stillschweigend:

Schreib- und Druckfehler.

Falsche Zahlen der Zitate.

Text der Zitate, wenn er ohne erkennbare Absicht von der Uberlieferung abweichend gegeben war. Beruhten jedoch die Ausfüirungen von Wilamowitz auf von der Uberlieferung versehentlich abweichendem Text, so wurde der Tatbestand durch gekennzeichnete Zusätze aufgeklärt.

Veraltete deutsche Orthographie. Die Regelung erfolgte durch die Druckerei.

Bemerkungen und Zusătze der Bearbeiter.

Die gekennzeichneten (s. oben) Bemerkungen und Zusätze der Bearbeiter betreffen hauptsächlich:

Bibliographische Angaben, wenn die von Wilamowitz gewählte Fassung entweder schwer verständlich oder, etwa angesichts neuer Ausgaben, unpraktisch geworden war. Zitate, deren Kürze trotz der durch diese entstehenden Schwierigkeiten beabsichtigt schien, blieben unverändert.

Verweise auf Behandlung des gleichen Gegenstandes an anderer Stelle der Schriften von Wilamowitz.

Die Verteilung der Bemerkungen und Zusätze auf Text, Anmerkungen und Anhang geschah je nach Lage des einzelnen Falles.

Ausgeschlossen wurde auf das strengste jede Kritik an den Ausführungen von Wilamowitz, auch in den Fallen, in denen Wilamowitz heute sicherlich selbst geändert hätte. 
